\title{
Modelling capillary break-up of particulate suspensions
}

Claire Mcllroy, and Oliver G. Harlen

Citation: Physics of Fluids 26, 033101 (2014); doi: 10.1063/1.4866789

View online: http://dx.doi.org/10.1063/1.4866789

View Table of Contents: http://aip.scitation.org/toc/phf/26/3

Published by the American Institute of Physics

\section{Articles you may be interested in}

Capillary breakup of suspensions near pinch-off

Physics of Fluids 27, 093301 (2015); 10.1063/1.4930011

Inhomogeneity in breakup of suspensions

Physics of Fluids 27, 063303 (2015); 10.1063/1.4922582

An experimental study of particle effects on drop formation

Physics of Fluids 16, 1777 (2004); 10.1063/1.1691034

An experimental study of drop-on-demand drop formation

Physics of Fluids 18, 072102 (2006); 10.1063/1.2217929

Drop formation and breakup of low viscosity elastic fluids: Effects of molecular weight and concentration

Physics of Fluids 18, 043101 (2006); 10.1063/1.2190469

Elasto-capillary thinning and breakup of model elastic liquids

Journal of Rheology 45, 115 (2000); 10.1122/1.1332389

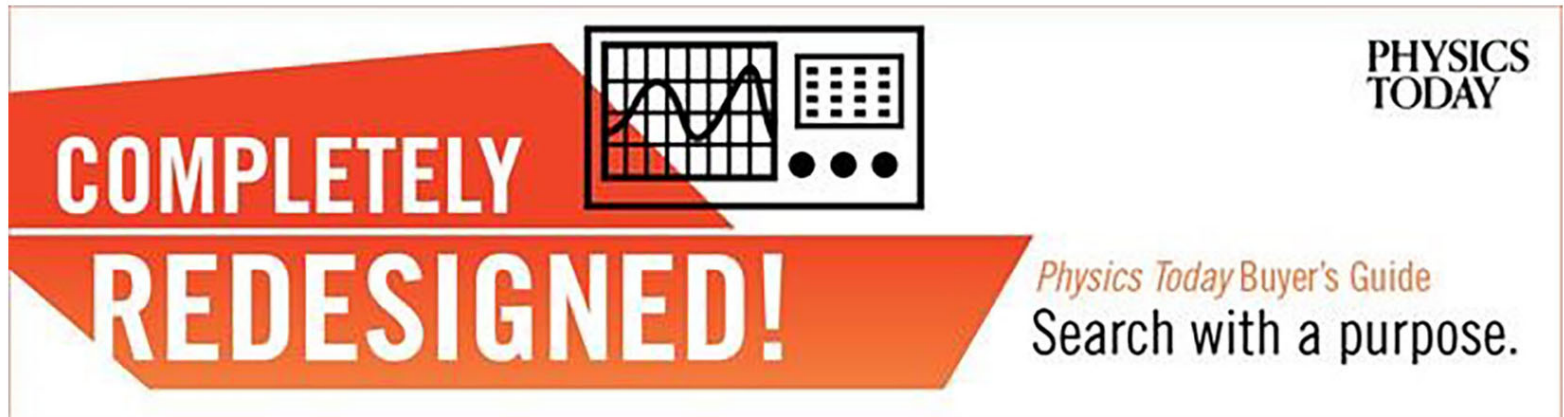




\title{
Modelling capillary break-up of particulate suspensions
}

\author{
Claire Mcllroy ${ }^{\mathrm{a})}$ and Oliver G. Harlen ${ }^{\mathrm{b})}$ \\ Department of Applied Mathematics, University of Leeds, Leeds LS2 9JT, United Kingdom
}

(Received 7 November 2013; accepted 8 February 2014; published online 3 March 2014)

\begin{abstract}
We have constructed a simple one-dimensional model of capillary break-up to demonstrate the thinning behaviour of particulate suspensions previously observed in experiments. The presence of particles increases the bulk viscosity of a fluid and so is expected to retard thinning and consequently delay the time to break-up. However, experimental measurements suggest that once the filament thins to approximately five particle diameters, the thinning no longer follows the behaviour predicted by the bulk viscosity; instead thinning is "accelerated" due to the effects of finite particle size. Our model shows that accelerated thinning arises from variations in local particle density. As the filament thins, fluctuations in the local volume fraction are amplified, leading ultimately to particle-free sections in the filament. The local viscosity of the fluid is determined from the local particle density, which is found by tracking individual particles within the suspension. In regions of low particle density, the fluid is less viscous and can therefore thin more easily. Thus, we are able to model the accelerated thinning regime found in experiments. Furthermore, we observe a final thinning regime in which the thinning is no longer affected by particle dynamics but follows the behaviour of the solvent. (C) 2014 AIP Publishing LLC. [http://dx.doi.org/10.1063/1.4866789]
\end{abstract}

\section{INTRODUCTION}

Inkjet printing is fast becoming a versatile, widely used manufacturing tool. A diverse range of materials can be manipulated using inkjet technology, the most common being particulate suspensions. Most graphical inks contain solid pigment particles rather than dyes and in many non-graphical applications, such as printed electronics, the functional components of the ink are solid particles. Solid-laden inks are also required in ceramic manufacture and textile printing. However, there is relatively little known about particulate effects on the stability and break-up of liquid jets compared to Newtonian fluids or even other complex fluids such as polymer solutions.

The detachment dynamics for simple Newtonian fluids are now well understood. ${ }^{1}$ Surface tension acts to destabilise a liquid jet, driving the free surface to minimise its surface energy and break up into spherical droplets. Viscous forces resist this thinning action and enable the liquid to be drawn into thin, uniform filaments before ultimately breaking up. Eggers ${ }^{2}$ developed the theory for the universal pinch off of an axisymmetric free surface, which states that the decay of a Newtonian jet depends on a balance of surface tension $\gamma$, inertia, and viscosity $\mu$, such that the minimum filament radius obeys the thinning law

$$
h_{\min }=0.0304 \frac{\gamma}{\mu}\left(t-t_{b}\right)
$$

where $t_{b}$ denotes the break-up time. This thinning law applies to cases of moderate Ohnesorge number, which is defined as

$$
O h=\frac{\mu}{\sqrt{\rho \gamma R}},
$$

\footnotetext{
a)Electronic mail: mm07c2m@leeds.ac.uk

b)Electronic mail: o.g.harlen@leeds.ac.uk
} 
where $\rho$ is the fluid density and $R$ is the jet radius. At extreme values of the Ohnesorge number, viscosity or inertia may dominate the dynamics giving different thinning laws. For highly viscous fluids with large Ohnesorge numbers, inertia may be neglected and Papageorgiou ${ }^{3}$ determined the thinning law

$$
h_{\min }=0.0709 \frac{\gamma}{\mu}\left(t-t_{b}\right),
$$

whereas for inertially dominated liquids the thinning may be described by ${ }^{4}$

$$
h_{\min }=0.64\left(\frac{\gamma}{\rho}\right)^{1 / 3}\left(t-t_{b}\right)^{2 / 3} .
$$

Both the inertial and viscous regimes eventually cross over to the universal Eggers solution at some critical radius. ${ }^{1}$ In the case of inertial flow, as the filament radius becomes thinner, the local Ohnesorge number increases so that eventually viscous effects are significant. On the other hand, inertia becomes surprisingly important near to break-up for viscosity dominated flows. In each case the local balance induces a transition to the Eggers regime (1) in which all three forces are significant.

Campo-Deano et al. ${ }^{5}$ propose that the critical Ohnesorge number defining the boundary between viscosity and inertia dominated regimes is

$$
O h^{*}=0.2077,
$$

with excellent agreement to their experimental results. Thus, for fluids with $O h<O h^{*}$ behaviour is initially governed by inertial forces, whereas a viscosity dominated regime is observed for fluids such that $O h>O h^{*}$. Ultimately, thinning adopts the universal Eggers regime (1), however, the critical radius at which this transition occurs may not be within observable limits. For inkjet printing applications, stable drop generation for Newtonian fluids is limited to a narrow range of viscosities ${ }^{6,7}$ corresponding roughly to Ohnesorge numbers in the range $0.1<O h<1$. If the Ohnesorge number is too high, then viscous forces prevent break off of the ligament from the nozzle. On the other hand, if the Ohnesorge number is too low, then surface tension causes the trailing ligament to break up into a number of unwanted satellite drops.

The existence of universal thinning laws initiated the use of capillary break-up as a rheological technique, allowing direct access to the viscosity of the fluid. Stretching a liquid sample between two end plates induces a strong extensional flow and thus allows the study of viscous properties under extensional deformation. Capillary break-up experiments have proved effective in measuring the extensional properties of polymeric fluids, such as relaxation time and extensional viscosity. ${ }^{5,8,9}$ Furthermore, recent studies have demonstrated that extensional rheometry can be successfully performed on particulate suspensions. ${ }^{10-12}$ However, the detachment dynamics of particle-laden fluids are not yet fully understood.

The presence of particles in a solvent increases the bulk viscosity of a fluid. Thus, particles are expected to retard the thinning process and consequently delay the time to break-up. However, experimental measurements using the pendant-drop technique ${ }^{13-15}$ suggest that once the filament has thinned to approximately five particle diameters, the thinning no longer follows the behaviour predicted by the bulk viscosity. In fact, the thinning is "accelerated" due to the effects of finite particle size. Furthermore, the thinning dynamics of particulate fluids are found to be less predictable than those of continuous fluids.

In particular, experiments on non-colloidal particle suspensions show a faster thinning rate than that predicted by the Papageorgiou regime for the bulk viscosity. For concentrated particle suspensions $\phi_{a v} \geq 20 \%$, Bonnoit et al. ${ }^{13}$ claim that this accelerated regime is independent of the initial volume fraction and can be described as the thinning of a viscous fluid with viscosity equal to that of a suspension of volume fraction 17\%. However, for inkjet printing purposes the solutions are generally more dilute. For smaller volume fractions $\phi_{a v}<6 \%$, van Deen et al. ${ }^{16}$ show that even the presence of a single particle in the thinning filament modifies the detachment dynamics, again accelerating the thinning rate.

A mathematical model has recently been developed to study the dynamics of filament evolution towards break-up in the presence of an embedded, solid, spherical particle. ${ }^{17}$ A single particle is 
modelled as a "stresslet" and assumed to remain stationary at the jet midpoint. Initially the jet evolves much like a pure liquid jet, until stretching-induced stresslet flow begins to alter the dynamics. This particle induced flow causes strong local deformation of the free surface and their results demonstrate the formation of a liquid bulge around the particle, with the point of pinch off shifting from the centre to accommodate the particle. Their results explain the observations in experiments ${ }^{14}$ that if a large enough number of particles become trapped in the filament region during the thinning process, then thinning is resisted rather than accelerated. The properties of particles suspended within a thinning filament are found to be critical to the stability of a liquid jet. However, this model does not explain the accelerated dynamics that is generally observed in experiments of particulate suspensions.

Our hypothesis is that the accelerated thinning regime arises from variations in the local particle density. As the filament thins, the variations are amplified leading ultimately to sections of the filament containing no particles at all. Sections of the filament that have a low particle density consequently have a lower viscosity and can therefore thin more easily. Similar fluctuations in particle density have been observed by Roche $e t a l .{ }^{18}$ during the thinning of a liquid bridge; close to break-up, certain regions within the bridge become jammed whilst particles experience a significant flow in other areas. To test our hypothesis, we have constructed a simple one-dimensional model of capillary break-up in which the viscosity is determined from the local particle density, found by tracking individual particles within the suspension. The particles are assumed to be non-Brownian so that they are simply advected with the fluid velocity. Since the particles only contribute to the dynamics through the local viscosity, the direct effects of hydrodynamic interactions between the particles and the effects of the individual particles on the shape of the free surface are not included. Nevertheless, our model is able to reproduce the accelerated thinning found in experiments.

\section{A ONE-DIMENSIONAL MODEL}

\section{A. Modelling capillary break-up}

We shall consider the thinning and break-up of a liquid bridge held between two end plates, as used in capillary break-up experiments. ${ }^{5}$ With the exception of the boundary conditions applied at the ends, this is equivalent to the break-up of an infinite jet and so local thinning behaviour is expected to be the same. We assume that the liquid bridge is long and thin so that we can treat it as a slender, axisymmetric jet. ${ }^{1}$

The slender-jet approximation assumes that velocity and stress are independent of the crosssectional area. We can therefore assume that the kinematics of the motion are one-dimensional and variables depend only on axial position $z$ and time $t$. A one-dimensional model is surprisingly accurate, even if the long-wavelength assumption is not well defined as in the liquid bridge problem. ${ }^{19,20}$ In their study of falling particle plumes, Crosby and Lister ${ }^{21}$ conclude that the effect of particle density modes in the $r$ and $\theta$ directions are unimportant. Thus, we shall assume that particle density varies only with the axial coordinate $z$.

Denoting the jet radius $h(z, t)$ and the velocity $v(z, t)$, we have the following governing equations, as derived by Forest and Wang. ${ }^{22}$ Conservation of mass yields

$$
\frac{\partial h^{2}}{\partial t}+\frac{\partial}{\partial z}\left(h^{2} v\right)=0
$$

and conservation of momentum is given by

$$
\frac{\partial}{\partial t}\left(h^{2} v\right)+\frac{\partial}{\partial z}\left(h^{2} v^{2}\right)=\frac{\partial}{\partial z}\left(h^{2}\left(K+3 O h \frac{\partial v}{\partial z}\right)\right),
$$

where the curvature term is defined as ${ }^{23}$

$$
K=\frac{h_{z z}}{\left(1+h_{z}^{2}\right)^{3 / 2}}+\frac{1}{h\left(1+h_{z}^{2}\right)^{1 / 2}},
$$

for the first $h_{z}$ and second $h_{z z}$ derivatives of $h$. Here the governing equations have been nondimensionalised using the Rayleigh time scale $T_{R}=\sqrt{\rho R_{0}^{3} / \gamma}$ for initial jet radius $R_{0}$. The 



FIG. 1. A one-dimensional model to represent the capillary thinning of a particulate suspension. Particles are sorted into "bins" of length $b z$. Shaded particles are allocated to a bin according to their position.

dimensionless Ohnesorge number $O h$ is defined for initial jet radius $R_{0}$ and describes the importance of the resisting viscous forces compared to the driving surface tension.

To simulate a capillary break-up experiment, we assume Dirichlet boundary conditions such that the free surface $h$ is fixed and there is zero axial velocity $v$ at the end-plates. The end-plates have radius $2 \mathrm{~mm}$ and are held at fixed separation distance $6 \mathrm{~mm}$. The initial shape of the free surface is modelled as an arc of a circle, with mid-filament radius set to $R_{0}=1 \mathrm{~mm}$ to induce capillary thinning.

The governing equations (4) and (5) are solved via a Lax-Wendroff scheme, which is secondorder accurate in both time and space. The equations are solved on a uniform mesh for nodes $j=1, \ldots, J$. The mesh size $d z$ satisfies the Courant stability condition $v d t \leq d z$ for time step $d t$. The stability of this explicit method is also subject to the condition that the time step $d t$ must be less than the diffusion time $h^{2} / O h$, thus is restricted at high viscosities. ${ }^{24}$ Hence, for $O h>3$, we have used an implicit numerical scheme.

\section{B. Modelling particle motion}

Initially, particles are uniformly distributed at random locations throughout the fluid. We assume that the number of particles $N$ is much larger than the number of mesh nodes $J$. The initial particle positions $z_{p}$ for $p=1, \ldots, N$ are assigned as follows.

We define the accumulated volume $V(z)$ as

$$
V(z)=\pi \int_{0}^{z} h^{2}\left(z^{\prime}\right) d z^{\prime}
$$

A corresponding $V$ position is chosen for each particle from a uniform distribution on the interval $\left[0, V_{t o t}\right]$, where $V_{t o t}$ is the volume of the entire liquid bridge. Then, the corresponding value of $z_{p}$ is found by inverting equation (6). In subsequent motion, we assume that each particle moves with the axial velocity $v\left(z_{p}, t\right)$ obtained by linear interpolation between grid points. The distribution of particles is then determined from particle position. A diagram of particle motion within the liquid bridge is shown in Figure 1.

Brownian motion opposes the creation of particle density gradients, however, in our model, we assume that the particles are sufficiently large that Brownian motion is negligible. The importance of particle diffusion on the length scale of the particle radius $r$ is measured by the Peclet number,

$$
P e=\frac{6 \pi \mu_{s} \dot{\epsilon} r^{3}}{k_{B} T},
$$

for the Boltzman constant $k_{B}$ and absolute temperature $T$. The characteristic stretching rate $\dot{\epsilon}$ is defined by the time scale of the flow, which is taken to be the smaller of the inverse Rayleigh time scale $\sqrt{\gamma / \rho R_{0}^{3}}$ or the inverse viscous time scale $\gamma / \mu R_{0}$. For an extension rate of $\dot{\epsilon} \sim 10 \mathrm{~s}^{-1}$, which is typical of capillary thinning experiments, the Peclet number for $1 \mu \mathrm{m}$ particles is of the order $\mathrm{Pe} \sim 10^{4}$ for solvent viscosity $389 \mathrm{mPa}$ s and filament radius $1 \mathrm{~mm}$. Thus, the large Peclet 

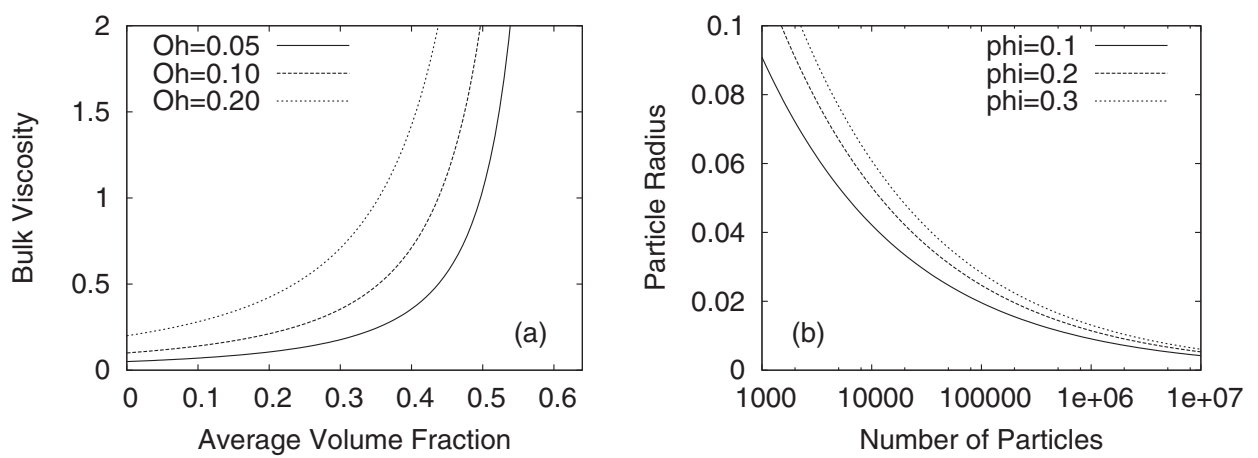

FIG. 2. (a) Plot showing the bulk viscosity $O h_{a v}$ obtained from the Krieger-Dougherty model as a function of particulate volume fraction for various values of solvent viscosity $O h_{s}$. (b) Effective particle radius $r$ as a function of the total number of particles $N$ for various volume fractions $\phi_{a v}$.

number assumption is valid for the pendant drop experiments, ${ }^{13,14}$ where the particle sizes are around $\sim 40-250 \mu \mathrm{m}$. For inkjet printing applications, where inks are much less viscous (say $11 \mathrm{mPa}$ s) and typical nozzle length scales are around $R_{0} \sim 50 \mu \mathrm{m}$, the extension rates are around $\dot{\epsilon} \sim 10^{4} \mathrm{~s}^{-1}$ and the limiting particle size is approximately $\sim 10 \mathrm{~nm}$.

\section{Local viscosity of suspensions}

The average volume fraction of particles in a suspension is given by

$$
\phi_{a v}=\frac{N}{V_{t o t}} V^{p},
$$

where $V^{p}$ is the particle volume, which for spherical particles is

$$
V^{p}=\frac{4}{3} \pi r^{3} .
$$

Thus, particle size can be varied by changing the total number of particles $N$ for a given average volume fraction $\phi_{a v}$.

We determine the local particle volume fraction by dividing the filament into a number of "bins," as shown in Figure 1. The length of each bin $b z$ is set to $\sim 2 r$ so that the length scale for volume fraction perturbations is set equal to the particle diameter. This bin size is typically larger than the grid resolution used in the velocity calculation.

The local viscosity is determined by the Krieger-Dougherty model ${ }^{25}$ so that the local Ohnesorge number in Eq. (5) is given by

$$
O h_{i}=O h_{s}\left(1-\frac{\phi_{i}}{\phi_{\max }}\right)^{-2}, \quad i=1, \ldots J,
$$

for local volume fraction $\phi_{i}$ and solvent Ohnesorge number $O h_{s}$. Here $\phi_{\max }$ is the maximum packing coefficient, which is found from numerical simulations to be $\phi_{\max }=0.64$ for random close-packing of monodisperse spheres. ${ }^{26}$ This maximum packing condition implicitly constrains the number of particles that can occupy a particular axial position. For a uniform distribution of particles, the average or bulk viscosity $O h_{a v}$ can be calculated by evaluating the Krieger-Dougherty model at the average volume fraction $\phi_{a v}$.

The concentration dependence of the bulk viscosity predicted by the Krieger-Dougherty model is plotted in Figure 2 for a range of solvent viscosities $O h_{s}$. Also shown in Figure 2 is the dependence of particle size on the total number of particles for a range of average volume fractions. For example, a suspension of $\phi_{a v}=20 \%$ particles will increase a solvent viscosity of $O h_{s}=0.1$ to the bulk value $O h_{a v}=0.22$ and for $N=100000$ particles the effective particle radius is $r=0.024$. 

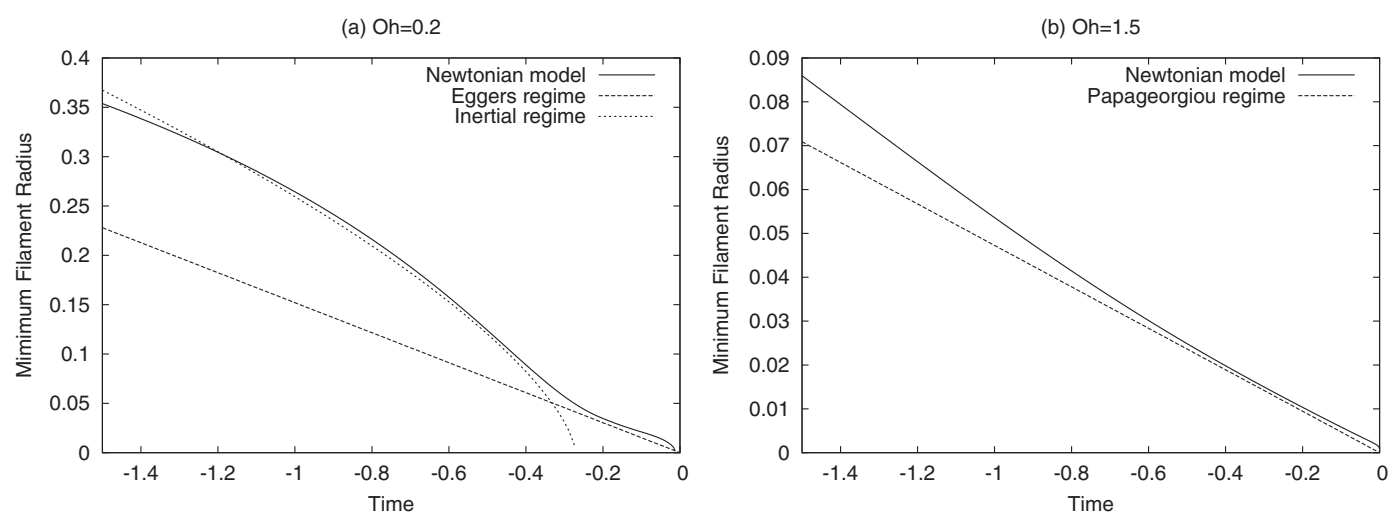

FIG. 3. (a) Newtonian model $O h=0.2$ for filament thinning compared to the inertial regime (3) and the Eggers regime (1). (b) Newtonian model $\mathrm{Oh}=1.5$ compared to the Papageorgiou viscous regime (2).

\section{RESULTS}

\section{A. Newtonian behaviour}

As discussed in the Introduction, the thinning dynamics of a liquid bridge are governed by a balance of surface tension, viscosity, and inertia characterised by the Ohnesorge number. Under the action of surface tension the liquid bridge develops into two hemispherical drops connected by a thin filament. In Figure 3 we compare the evolution of the minimum filament radius predicted by our model for two Newtonian fluids of differing viscosities.

In Figure 3(a) we show the results for a fluid with $O h=0.2$ (typical of inkjet printing fluids for which $0.1<O h<1$ ), where the origin of time is shifted to the break-up time. Since $O h<O h^{*}$ the thinning is initially dominated by inertia and is seen to follow the inertial regime given by Eq. (3). The Ohnesorge number of this Newtonian fluid is close to the critical value $O h^{*}=0.2077$, thus we are able to observe the transition to the universal Eggers regime given by Eq. (1). This transition occurs when the filament radius has thinned to approximately $h_{\min } \sim 0.05$.

In contrast, a fully developed viscous regime is seen for $O h=1.5$ in Figure 3(b), where the radial decay follows Papageorgiou's linear thinning regime (2). These results are in agreement with the experimental observations of Campo Deano et al. ${ }^{5}$

\section{B. The effect of particles on thinning behaviour}

The addition of particles to a Newtonian solvent increases the bulk viscosity of the fluid, as predicted by the Krieger-Dougherty equation (7). For solvent viscosity $O h_{s}=0.1$, the addition of $\phi_{a v}=20 \%$ particles to the Newtonian solvent increases the viscosity to the bulk value $O h_{a v}=0.22$. The increased resistance acts to retard the thinning process and Figure 4 shows that the time to breakup for a Newtonian fluid with the bulk viscosity is nearly twice as long as that of the solvent viscosity.

Figure 4 also shows the mean radial decay profile of ten realisations predicted by our particle model, where the effective particle size is $r=0.024$ relative to the initial mid-filament radius; the shaded region indicates the standard deviation from the mean. The particulate suspension is seen initially to follow the behaviour of the bulk viscosity. However, as the filament radius decays we observe accelerated thinning and the time to break-up is consequently reduced. The results of choosing different bin sizes are shown in Figure 5 and compared to the bulk viscosity model. The difference in the radial decay profile for different bin lengths is small compared to the effect of particles on the bulk behaviour. Furthermore, the difference due to changing bin size is within the standard deviation measured for the suspension shown in Figure 4. Consequently, the specific bin size does not significantly affect the dynamics provided $b_{z}<2 r$ but remains small compared to the filament length. 


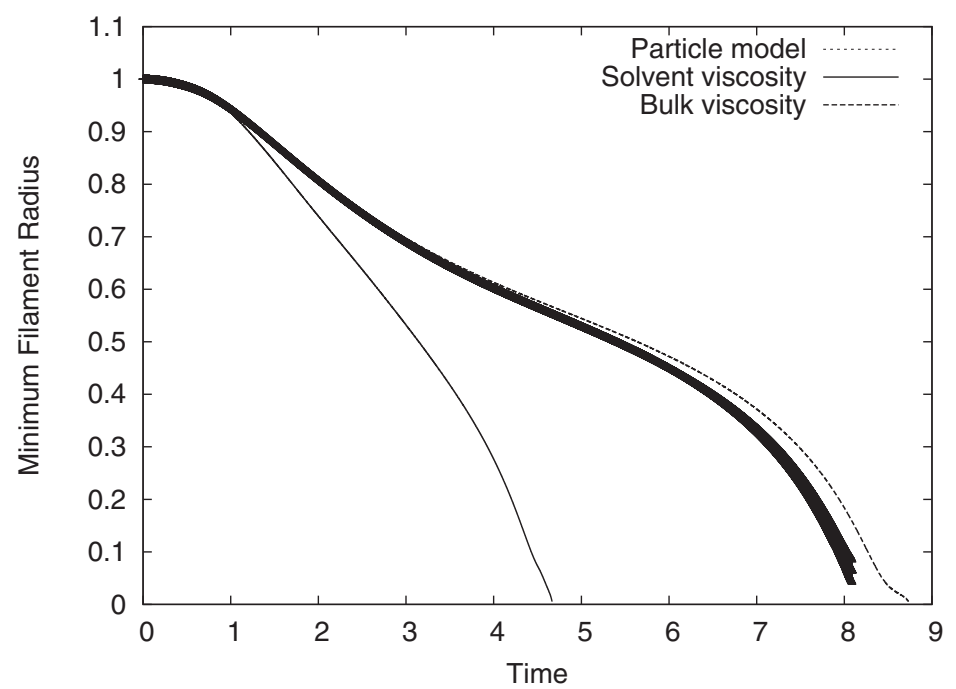

FIG. 4. Radial decay profiles of pure solvent $O h_{s}=0.1$, bulk viscosity $O h_{a v}=0.22$, and the corresponding particulate suspension of volume fraction $\phi_{a v}=20 \%$ and particle size $r=0.024$. The shaded area shows that standard deviation from the mean for ten realisations.

To understand particle effects on the thinning behaviour, we study the evolution of the local particle density. Since the particles move with the fluid velocity, they are transported out of the thinning filament and into the end drops as the filament radius decays. Thus, although the average particle density in the filament remains constant, large fluctuations in the local particle density appear, as shown in Figure 6(a). Near to break-up, there are regions deplete of particles as well as regions of high density.

Figure 6(b) shows that the particle fluctuations are mirrored in the fluid viscosity so that there are regions of both high and low viscosity in the filament compared to the bulk viscosity $O h_{a v}=0.22$. The viscosity is reduced to that of the solvent $O h_{s}=0.1$ in areas devoid of particles. It is these areas of low viscosity that allow the filament to neck and thin faster than a Newtonian fluid of the bulk viscosity.

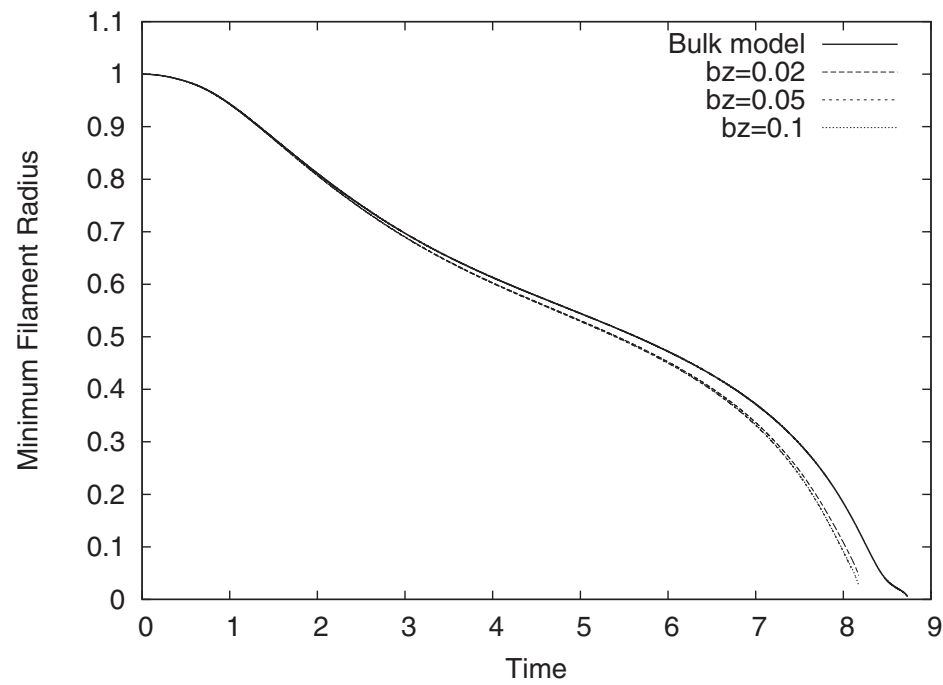

FIG. 5. Radial decay profile of a particle suspension for varying bin size $b z$ compared to the bulk viscosity model for suspension properties $O h_{s}=0.1, \phi_{a v}=20 \%, r=0.024$. 

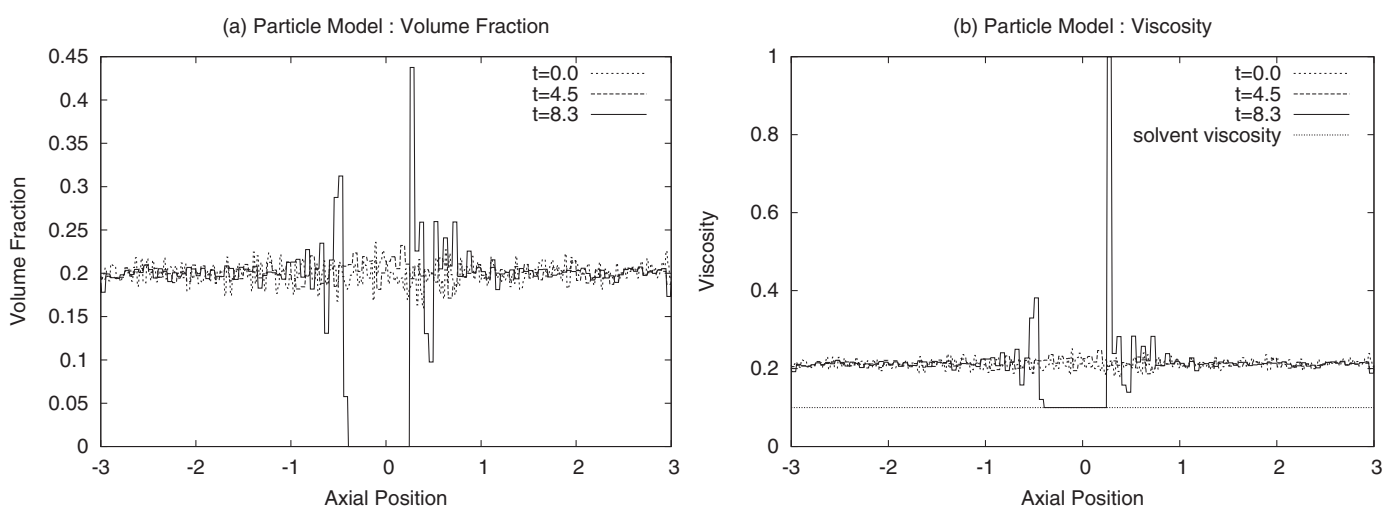

FIG. 6. (a) Volume fraction evolution profile and (b) viscosity evolution profile for suspension properties $O h_{s}=0.1$, $\phi_{a v}=20 \%, r=0.024$

The free surface evolution profile of the particulate suspension is shown in Figure 7(a). Although our model is unable to predict individual particle effects on the free surface, close to break-up the profile appears "lumpy" with variations in filament thickness that reflect variations in particle concentration. Particle-rich regions appear as bulges that correspond to clusters of particles. In the region containing no particles, the free surface is able to thin down and form a uniform filament, as observed in the corresponding Newtonian case shown in Figure 7(b).

A plot of particle evolution with time is shown in Figure 8, which provides a spatio-temporal diagram of the variation in volume fraction with radial decay and axial position. We illustrate two different scenarios in Figures 8(a) and 8(b); first, we show the full particle model, where the onset of particle fluctuations is fed back into the fluid viscosity and therefore affects the local thinning dynamics; second, we show a hypothetical case in which viscous feedback is neglected and we consider only the motion of the particles in a fluid of uniform viscosity. The latter case is referred to as the average viscosity model. By comparing Figures 8(a) and 8(b), we can examine how the dynamics affect fluctuations in particle density.

In particular, Figure 8(a) shows how the volume fraction variations grow with time for one particular realisation of a suspension with $O h_{s}=0.1, \phi_{a v}=20 \%, r=0.024$ whereas Figure 8(b) shows how the fluctuations would have evolved if we hypothetically impose a uniform viscosity $O h=0.22$, which corresponds to the average volume fraction used in the full particle simulation. We continue by highlighting the differences between these two plots and describing how particle fluctuations develop as a consequence of variations in the local viscosity.
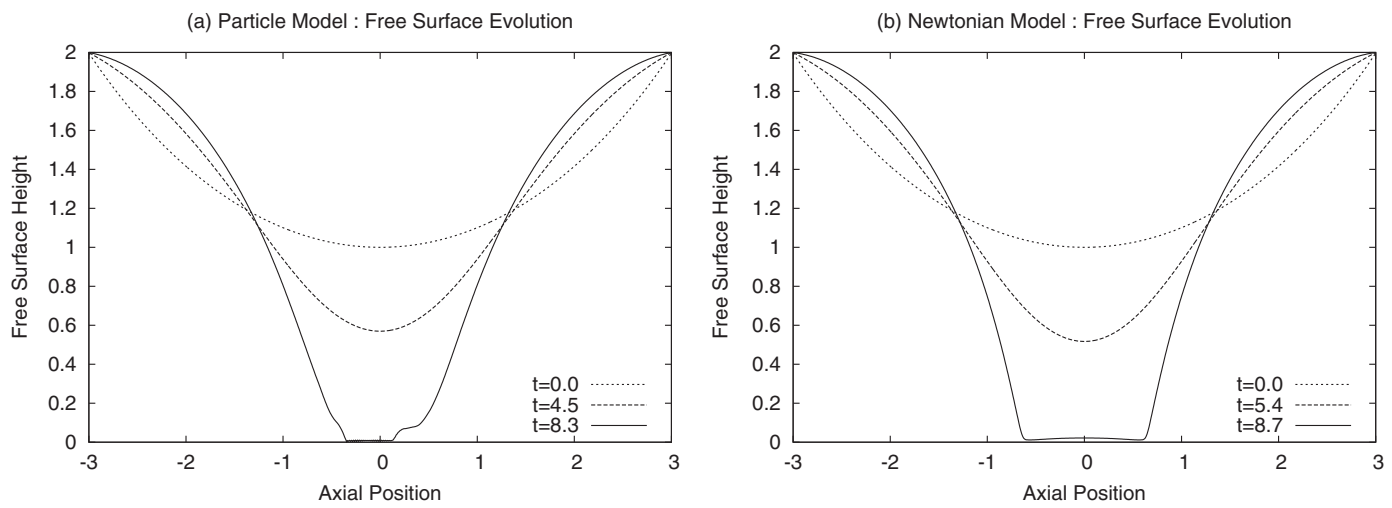

FIG. 7. Free surface evolution profile of (a) suspension with properties $O h_{s}=0.1, \phi_{a v}=20 \%, r=0.024$ and (b) bulk viscosity Newtonian fluid $O h_{a v}=0.22$. 



FIG. 8. (a) Evolution of volume fraction variations for one realisation in the case $O h_{s}=0.1, \phi_{a v}=20 \%, r=0.024$ predicted by the particle model. (b) Evolution of particle fluctuations for the same suspension properties but for a hypothetically imposed uniform viscosity $O h=0.22$ (see text for details). Each is compared to the free surface profile at the point of break-up.

In each case, the initial statistically uniform state corresponding to $h_{\min }=1$ shows little variation in the particle density. It is evident that fluctuations are amplified as the volume of fluid in the filament is reduced, which can be observed in the development of light and dark areas in Figure 8. For the average viscosity model shown in case (b), random clusters of particles form during radial decay, whereas the fluctuations in density develop much more smoothly for the full particle simulation shown in case (a).

In the latter case, the particle density feeds back into the fluid viscosity. The higher viscosity of particle-rich regions means that these tend not to thin further, but are advected along the filament. On the other hand, in the light areas that contain a lower particle density, the viscosity is reduced and consequently the region is able to thin more rapidly than the rest of the fluid. The reduction in volume drives more particles out of the filament into the end drops so that the low-viscosity region is able to develop into a uniform filament. We observe that, in most realisations, the viscous feedback of the particle model allows a single, uniform, low-viscosity filament to form between the two end drops. This is in contrast to the average viscosity model, which neglects dynamic feedback, where we see alternating regions of low and high volume fraction over the length of the filament. In our model, we neglect the effects of individual particles on the free surface. These effects are expected to become important only when the filament diameter is of the order of the particle diameter. However, at this point we observe that the filament contains no particles at all and consequently the local thinning dynamics at the minimum filament radius are unlikely to be affected by these interactions.

Other structures are possible depending on the initial distribution of particles, as shown in Figure 9. Instead of a single uniform filament, we observe an area of high particle density located at the centre of the axis, with regions deplete of particles surrounding it. This corresponds to the generation of a small satellite drop at the mid-filament point, which is connected to the end drops by threads of liquid. Instead of being advected into the end drops, particles remain trapped in the satellite indicated by the darker central region, which does not thin down due to its high local viscosity. The 

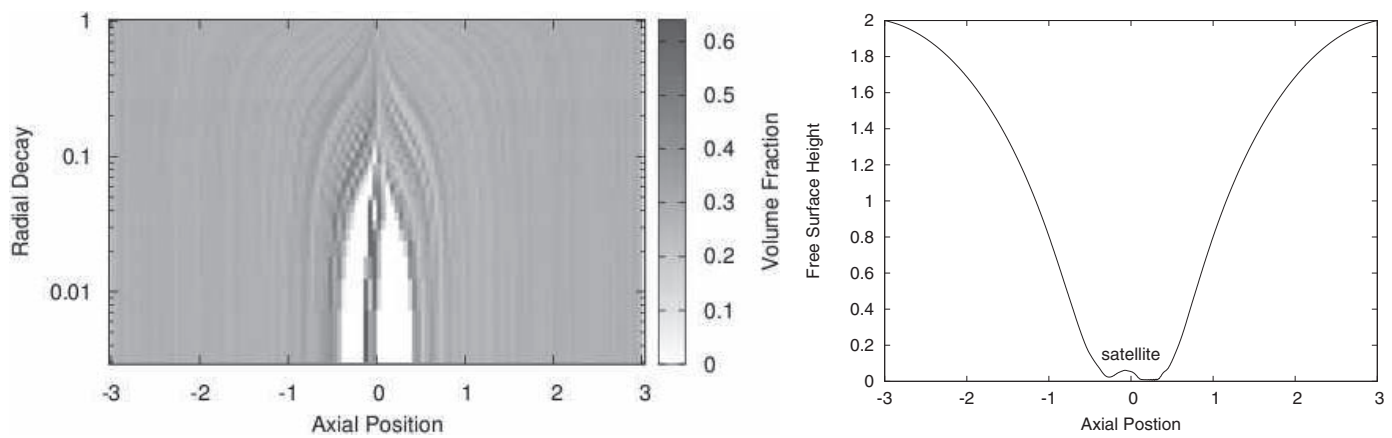

FIG. 9. Evolution of volume fraction fluctuations for one realisation predicted by the particle model compared to the free surface profile at the point of break-up for $O h_{s}=0.1, \phi_{a v}=20 \%, r=0.024$.

surrounding areas have a lower viscosity and are therefore able to thin more rapidly than the central droplet. This creates two shorter, uniform filaments that suspend the particle dense satellite drop between the end drops. Similar "beads-on-string" morphologies have been observed by Zimoch and McKinle ${ }^{27}$ and also compared to a one-dimensional model. For this "beads-on-string" case, the particle interactions with the free surface are more important and our model assumptions do not hold in the region of the bead. However, our model will still capture the differential thinning of the connecting filaments that contain no particles.

We have calculated the standard deviation of particle density from the average volume fraction $\phi_{a v}=20 \%$ and taken the mean over six realisations. Figure 10(a) compares the particle model to the average viscosity model without dynamics and Figure 10(b) highlights the difference between the two cases. The variation in particle fluctuations is equivalent in the initial stages of thinning. The effect of viscosity variation on the distribution becomes evident at approximately $h_{\min } \sim 0.1$, where the fluctuations observed in the particle model grow more slowly than in the case without viscosity feedback. In this example the dimensionless particle radius is calculated to be $r=0.024$. Thus, the point at which finite size affects the dynamics occurs when the filament radius has reduced to around four-five times the particle radius. This observation is not affected by the bin discretisation, as we have shown in Figure 5. This change in dynamics agrees with experimental observations that the dynamics follow that of the bulk viscosity up to the point where the filament diameter has thinned to approximately five particle diameters. ${ }^{15}$

Figure 11 shows the effect of increasing particle size on the mean minimum filament radius and the time to break-up. As particle size increases, the total number of particles in the simulation decreases and as a consequence the statistical variability between realisations increases. Therefore, more realisations are required in order to determine the mean break-up time. We observe that the
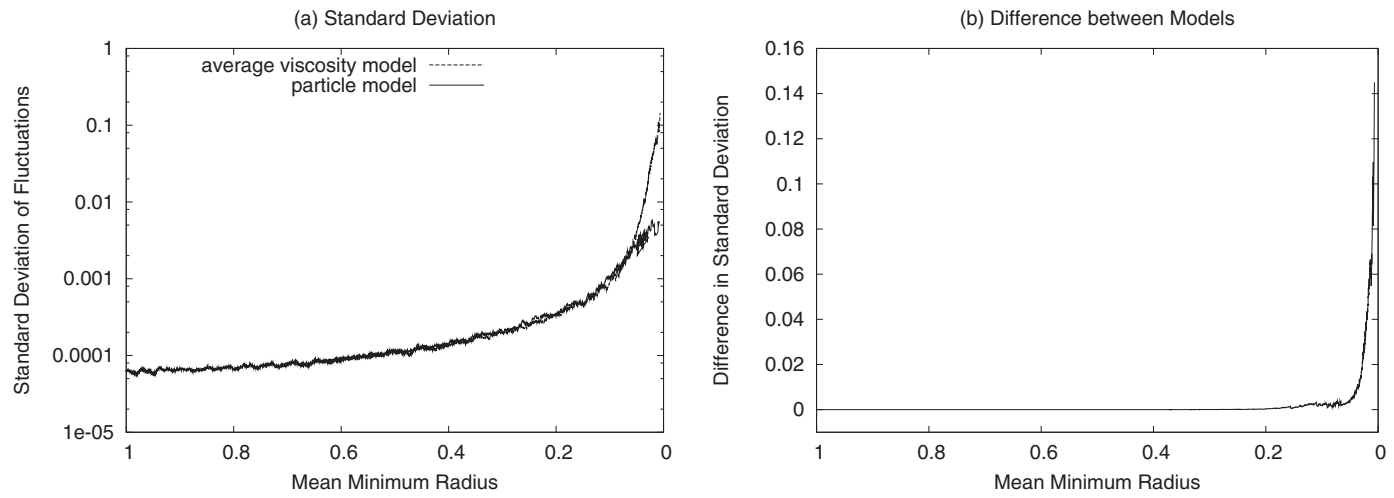

FIG. 10. (a) Standard deviation of particle density from the average volume fraction $\phi_{a v}=20 \%$ averaged over six realisations for suspension properties $O h_{s}=0.1, r=0.024$. (b) Difference between the particle model and average viscosity model. 


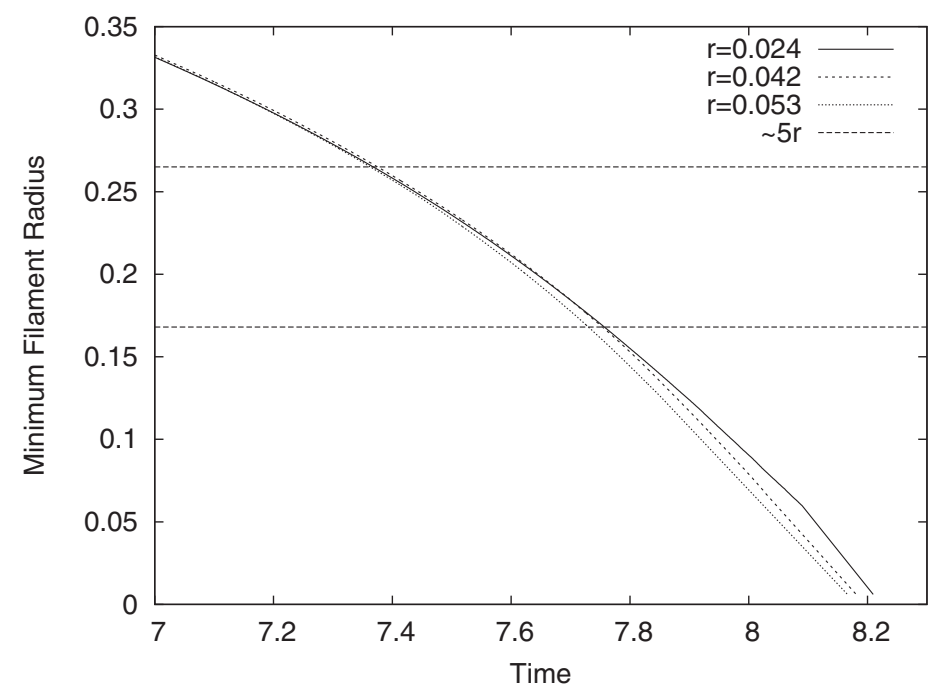

FIG. 11. Radial decay profile of a particulate suspension $O h_{s}=0.1, \phi_{a v}=20 \%$ for varying particle size.

thinning behaviour is equivalent for each particle radii until finite size affects the dynamics at approximately $h_{\min } \sim 5 r$. For larger particles, fluctuations in volume fraction are amplified and these variations occur earlier in the thinning process. Thus, increasing particle size further reduces the average time to break up. For very small particles $r \ll 0.01$, Newtonian behaviour will be recovered.

For a range of Ohnesorge numbers, volume fractions, and particle sizes, we find that the mean time to break-up of a particulate suspension is reduced in each case, in comparison with the break-up time of the corresponding Newtonian fluid of the bulk viscosity. However, variation from the mean break-up time is found to increase with each of these properties. The values of the standard deviation normalised by the mean break-up time, averaged over ten realisations, are shown in Figure 12. Hence, as seen in experiments, the behaviour of particulate suspensions is less predictable than that of the equivalent continuum fluid.

Although on average the time to break-up for a particulate suspension is decreased compared to that of the continuous fluid, this may not necessarily be the case for a single realisation. Our model demonstrates that as particle size and volume fraction are increased, the standard deviation from the mean break-up time is increased. The spread of break-up times depends upon the distribution of particles as break up approaches. In general a particle-free filament, having a lower viscosity than the bulk, is generated and we observe accelerated thinning due to particle effects. However, in some cases particles become trapped in the filament region creating areas of high viscosity compared to
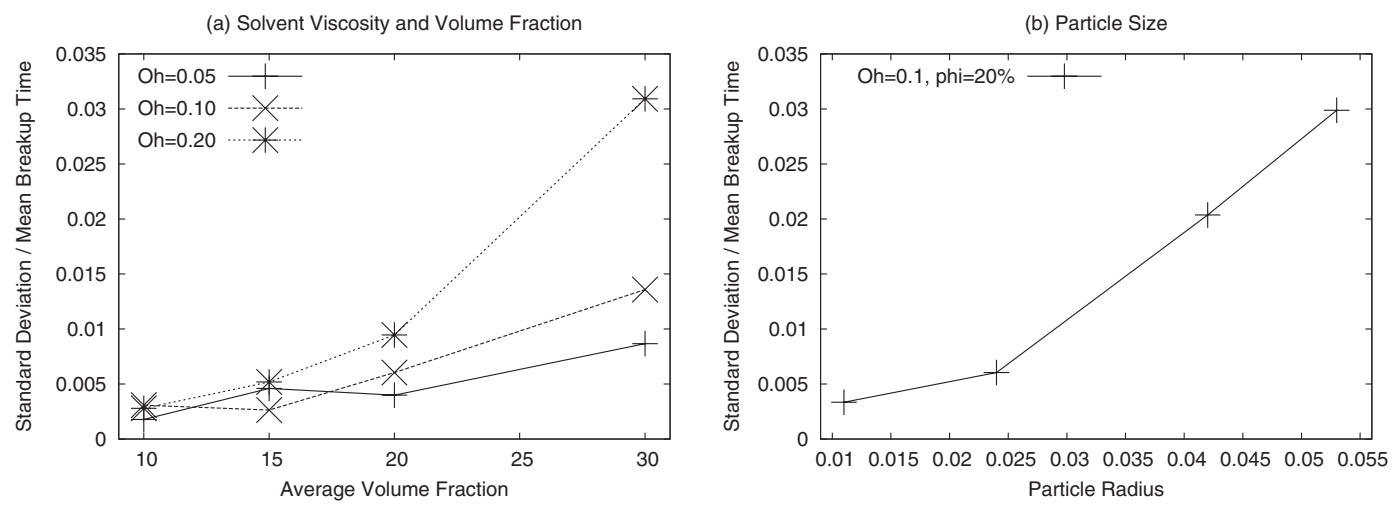

FIG. 12. Standard deviation divided by mean break-up time averaged over ten realisations. (a) Increasing solvent viscosity $O h_{s}$ and volume fraction $\phi_{a v}$. (b) Increasing particle size. 
the bulk. These structures may change the whole pinch off dynamics depending on the number of particles that are trapped in the filament.

Experiments have shown that, for small numbers of particles in the filament, finite size effects induce accelerated thinning as discussed above. On the other hand, if a sufficiently large number of particles become trapped in the filament region, then the effect tends to be stabilising; the individual motions of the particles are restricted and the necking of the filament is slowed. ${ }^{14}$ Hammeed and Morris ${ }^{17}$ study this mechanism further by modelling the local deformation of the fluid interface due to particle-induced flow. They show that for a single particle located at the centre of a thinning filament, the thinning rate is decreased for larger particle sizes. Consequently, the larger the particle trapped in the filament, the more stable the filament is and break-up time is consequently delayed.

Our model does not include this stabilisation mechanism, which occurs at a later stage in the development when the minimum filament radius is less than the particle radius. However, our model does predict that thinning becomes more varied as particle size and volume fraction are increased. Fluctuations in the particle density become more random and so it is more likely that more particles become trapped in the filament for highly concentrated suspensions containing larger particles. Thus, the stabilising effect of increasing particle size or volume fraction may decrease the number of satellite drops produced during jetting.

\section{Thinning regimes of particulate suspensions}

We have already observed that the initial thinning behaviour of a particulate suspension follows that of the corresponding Newtonian fluid of bulk viscosity. As the filament radius is reduced, the thinning enters an accelerated thinning regime due to finite size effects. For solvent viscosity $O h_{s}$ $=0.2 \mathrm{we}$ have tested a range of average volume fractions $\phi_{a v}=0.15,0.20,0.25$. The thinning profiles for each fraction collapse onto a single curve near the break-up point, as shown in Figure 13, where the origin of time has been shifted to the break-up time. This suggests that the thinning behaviour near to break-up is independent of the initial volume fraction $\phi_{a v}$. The accelerated regime we observe is not only faster than the rate of the corresponding Newtonian fluid of bulk viscosity, it is faster than the thinning rate of the pure solvent, which is also shown in Figure 13.

We have seen that as the filament becomes devoid of particles, the viscosity is reduced to that of the solvent. Thus, we would expect to observe a transition from the acceleration phase to a final regime in which the thinning follows the behaviour of the solvent. However, in these cases it is difficult to determine a final thinning regime adopted by the suspension in comparison to the solvent



FIG. 13. Radial decay profiles near to the point of break-up for a range of average volume fractions $\phi_{a v}=0.15,0.20,0.25$ compared to that of the solvent $O h_{s}=0.2$. 


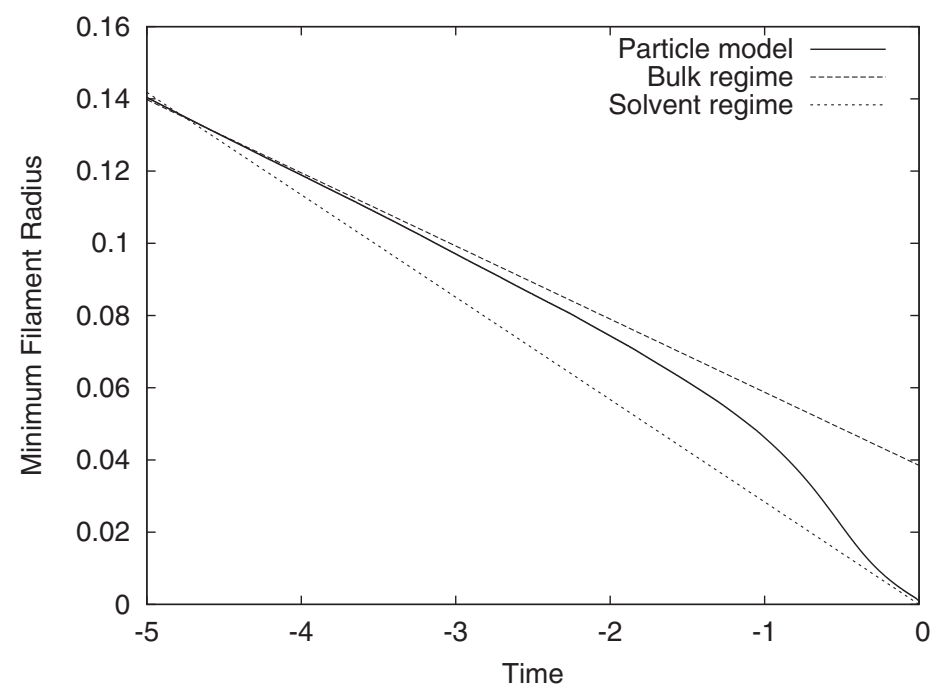

FIG. 14. Radial decay profile near to break-up for volume fraction $\phi_{a v}=10 \%$ and particle size $r=0.02$ compared to the Papageorgiou regime (2) for the solvent $O h_{s}=2.5$ and the bulk viscosity $O h_{a v}=3.5$.

behaviour since $O h_{s} \approx O h^{*}$. For Newtonian fluids having this Ohnesorge number, we observe a transition to the universal Eggers regime, which occurs at around the same radius that finite size affects the dynamics. Thus, at this low Ohnesorge number, inertia still plays a significant role in the thinning process and may obscure dynamics due to particle effects.

In order to avoid this, we consider a larger Ohnesorge number for which a fully developed Papageorgiou regime (2) is observed. Figure 14 illustrates the thinning profile of a particulate suspension with solvent viscosity equivalent to $O h_{s}=2.5$. For volume fraction $\phi_{a v}=10 \%$, the bulk viscosity increases to $O h_{a v}=3.5$ and for this suspension, the effective particle size is given by $r=0.02$.

The thinning profile of the suspension initially follows the bulk behaviour and Figure 14 shows that the radial decay obeys Papageorgiou's thinning law for the bulk viscosity (2). We then observe a transition to the accelerated thinning regime due to finite size effects at approximately $h_{\min }$ $\sim 5 r$; particles are forced into the end drops and particle-free regions develop. Again, the rate of the accelerated regime is seen to be faster than the thinning rate of the pure solvent given by Papageorgiou's law (2), which is valid for long, uniform filaments. Acceleration is attributed to the fact that a depleted filament is not yet slender, thus has a relatively high curvature and therefore must thin faster than a uniform filament in order to conserve volume. ${ }^{28}$ When the filament becomes sufficiently long and thin, the thinning dynamics enter a final regime. Figure 14 shows that the transition into this regime occurs at around $h_{\min } \sim r$ and the thinning behaviour can be described solely by the solvent properties, characterised by Papageorgiou's law for the solvent viscosity (2).

If large numbers of particles become trapped within the filament, then the generation of a slender filament may be restricted. Our model predicts this is more likely to occur for higher volume fractions. For these cases, we propose that the solvent regime will be observed once the filament has thinned to less than the particle radius. A liquid bridge of solvent is suspended between two individual particles, which subsequently forms a slender filament following Papageorgiou's law for the solvent viscosity. However, our model is valid only for $h_{\min }>r$, since we neglect individual particle effects on the free surface.

\section{CONCLUSION}

We have developed a simple one-dimensional model of capillary break-up to demonstrate experimental observations of drop formation from suspensions. A two-stage thinning model has previously been suggested ${ }^{14}$ based on the idea that the initial and final thinning dynamics involve a 
transition from a regime where particle effects are governed by bulk properties, to one where finite size effects dominate the behaviour.

For an initially uniform distribution of particles, our model predicts that as a liquid filament thins, fluctuations in the local particle density are amplified resulting in areas of both high and low particle density. These fluctuations are reflected in the fluid viscosity. Initially, the particulate suspension behaves like a Newtonian fluid with the corresponding bulk viscosity, however, the development of low-viscosity regions allows the filament to thin more easily. We therefore observe an accelerated thinning regime in which the thinning rate is faster than that of the bulk and the solvent behaviour. Furthermore, our model is able to quantify that finite size effects dominate when the filament radius has thinned to approximately five times the radius of the particle, as seen in large scale pendant-drop experiments. ${ }^{13-15}$

For high-viscosity suspensions, we observe a transition from the accelerated regime to an ultimate thinning regime once the depleted filament has become sufficiently long and thin. Since the filament is particle-free, the thinning is no longer governed by finite size effects but follows the behaviour of the pure solvent, which is characterised by Papageorgiou's thinning law. For lowviscosity suspensions, a slender filament is not generated at this final stage and the solvent regime is not observed.

\section{ACKNOWLEDGMENTS}

This work was supported by the UK Engineering and Physical Sciences Research (EPSRC) through Grant No. EP/H018913/1 Innovation in Industrial Inkjet Technology. We would like to thank Christian Clasen and Wouter Mathues from the University of Leuven for helpful discussions.

${ }^{1}$ J. Eggers and E. Villermaux, "Physics of liquid jets," Rep. Prog. Phys. 71, 036601 (2008).

2 J. Eggers, "Universal pinching of 3D axisymmetric free-surface flow," Phys. Rev. Lett. 71, 3458 (1993).

${ }^{3}$ D. T. Papageorgiou, "On the breakup on viscous liquid threads," Phys. Fluids 7, 1529-1544 (1995).

${ }^{4}$ R. F. Day, E. J. Hinch, and J. R. Lister, "Self-similar capillary pinchoff of an inviscid fluid," Phys. Rev. Lett. 80, 704 (1998).

${ }^{5}$ L. Campo-Deano and C. Clasen, "The slow retraction method (SRM) for the determination of ultra-short relaxation times in capillary breakup extensional rheometry experiments," J. Non-Newt. Fluid Mech. 165, 1688-1699 (2010).

${ }^{6} \mathrm{~B}$. Derby, "Inkjet printing of functional and structural materials: Fluid property requirements, feature stability and resolution," Annu. Rev. Mater. Res. 40, 395-414 (2010).

${ }^{7}$ G. H. McKinley and M. Renardy, "Wolfgang von Ohnesorge," Phys. Fluids 23, 127101 (2011).

${ }^{8}$ M. Tembely, D. Vadillo, and M. R. Mackley, "The matching of a 'one-dimensional' numerical simulation and experimental results for low viscosity Newtonian and non-Newtonian fluids during fast filament stretching and subsequent breakup," J. Rheol. 56, 159-183 (2012).

${ }^{9}$ M. Yao and G. H. McKinley, "Numerical simulations of extensional deformations of viscoelastic bridges in filament stretching devices," J. Non-Newt. Fluid Mech. 74, 47-88 (1998).

${ }^{10}$ E. E. Bischoff White, M. Chellamuthu, and J. P. Rothestein, "Extensional rheology of shear thickening cornstarch and water suspension," Rheol. Acta 49, 119-129 (2010).

${ }^{11}$ M. Chellamuthu, E. M. Arndt, and J. P. Rothestein, "Extensional rheology of shear thickening nano-particle suspensions," Soft Matter 5, 2117-2124 (2009).

${ }^{12}$ M. Smith, R. Besseling, M. E. Cates, and V. Bertola, "Dilatancy in the flow and fracture of stretched colloidal suspensions," Nat. Commun. 1, 114 (2010).

${ }^{13}$ C. Bonnoit, E. Clement, and A. Lindner, "Accelerated drop detachment in dense granular suspensions," Phys. Fluids 24, 043304 (2012).

${ }^{14}$ R. J. Furbank and J. F. Morris, “An experimental study of particle effects on drop formation," Phys. Fluids 16, 1777-1790 (2004).

${ }^{15}$ R. J. Furbank and J. F. Morris, "Pendant drop thread dynamics of particle-laden liquids," Int. J. Multiphase Flow 33, 448-468 (2007).

${ }^{16}$ M. S. van Deem, T. Bertrand, N. Vu, D. Quere, E. Clement, and A. Lindner, "Particles accelerate the detachment of viscous liquids," Rheol. Acta 52, 403-412 (2013).

${ }^{17}$ M. Hameed and J. F. Morris, "Breakup of a liquid jet containing solid particles: A singularity approach," SIAM J. Appl. Math. 70, 885-900 (2009).

${ }^{18}$ M. Roche, H. Kellay, and H. A. Stone, "Heterogeneity and the role of normal stresses during the extensional thinning of non-Brownian shear-thickening fluids," Phys. Rev. Lett. 107, 134503 (2011).

${ }^{19}$ B. Ambravaneswaran, E. D. Wilkes, and O. A. Basaran, "Drop formation from a capillary tube: Comparison of onedimensional and two-dimensional analysis and occurrence of satellite drops," Phys. Fluids 14, 2606-2621 (2002).

${ }^{20}$ J. Eggers and T. F. Dupont, "Drop formation in a one-dimensional approximation of the Navier-Stokes equation," J. Fluid Mech. 262, 205-221 (1994). 
${ }^{21}$ A. Crosby and J. R. Lister, "Falling plumes of point particles in viscous fluid," Phys. Fluids 24, 123101 (2012).

${ }^{22}$ M. G. Forest and Q. Wang, "Change-of-type behaviour in viscoelastic slender jet models," J. Theor. Comput. Fluid Dyn. 2, 1-25 (1990).

${ }^{23}$ V. M. Entov and A. L. Yarin, "Influence of elastic stress on the capillary breakup of dilute polymer solutions," Fluid Dyn. 19, 21-29 (1984).

${ }^{24}$ C. Clasen, J. Eggers, M. A. Fontelos, and J. Li, “The beads-on-string structure of viscoelastic threads," J. Fluid. Mech. 556, 283-308 (2006).

${ }^{25}$ I. M. Krieger and T. J. Dougherty, “A mechanism for non-Newtonian flow in suspensions of rigid spheres," Trans. Soc. Rheol. 3, 137-152 (1959).

${ }^{26}$ M. D. Rintoul and S. Torquato, "Computer simulations of dense hard-sphere systems," J. Chem. Phys. 105, 9258-9265 (1996).

${ }^{27}$ P. J. Zimoch and G. H. McKinley, “Capillary breakup of discontinuously rate thickening suspensions," Phys. Rev. Lett. 111, 036001 (2013).

${ }^{28}$ C. Clasen and W. Mathues, private communication (2013). 